06,13

\title{
Квазистационарные процессы диэлектрической релаксации в тонких поликристаллических пленках PZT
}

\author{
() В.В. Иванов ${ }^{1}$, Е.Н. Голубева ${ }^{1}$, О.Н. Сергеева ${ }^{1,2,}$, Г.М. Некрасова ${ }^{2}$, И.П. Пронин ${ }^{3}$, Д.А. Киселев ${ }^{4}$ \\ ${ }^{1}$ Тверской государственный университет, \\ Тверь, Россия \\ ${ }^{2}$ Тверская государственная сельскохозяйственная академия, \\ Тверь, Россия \\ ${ }^{3}$ Физико-технический институт им. А.Ф. Иофффе РАН, \\ Санкт-Петербург, Россия \\ ${ }^{4}$ Национальный исследовательский технологический университет „МИСиС“, \\ Москва, Россия \\ ฯ E-mail: o_n_sergeeva@mail.ru \\ Поступила в Редакцию 17 апреля 2020 г. \\ В окончательной редакции 17 апреля 2020 г. \\ Принята к печати 21 апреля 2020 г.
}

\begin{abstract}
Проведены исследования релаксационных процессов в поликристаллических пленках РZT, сформированных на кремниевых подложках при квазистатическом изменении внешнего электрического поля. Показано, что диэлектрическая релаксация характеризуется, по крайней мере, тремя временами релаксации, зависящими от направления самополяризации в пленке, величины поляризующего поля, а также от температуры отжига пленок PZT.
\end{abstract}

Ключевые слова: тонкие поликристаллические пленки РZT, ВЧ магнетронное распыление, релаксационные явления.

DOI: $10.21883 /$ FTT.2020.10.49916.093

Интерес к исследованию тонких пленок цирконататитаната свинца (PZT) как к наиболее значимым функциональным материалам в современной микроэлектронике, не ослабевает и в настоящее время. Использование их в качестве элементов энергонезависимой памяти $[1,2]$ обосновывает актуальность исследования их переключательных свойств, особенность которых заключается в наличии нескольких квазистационарных релаксационных процессов с различными временами релаксации, однако в настоящее время нет однозначного заключения об их физической природе [3-7]. Особенно это касается самого короткого времени релаксации, составляющего порядка нескольких секунд.

В настоящей работе изучение релаксационных явлений проводилось на поликристаллических пленках PZT, полученных методом ВЧ магнетронного распыления керамической мишени состава $\mathrm{PbZr}_{0.54} \mathrm{Ti}_{0.46} \mathrm{O}_{3}$, находящегося в области морфотропной фазовой границы, на платинированную кремниевую подложку при температуре $150^{\circ} \mathrm{C}$ и затем, для образования фазы перовскита, подвергались отжигу (кристаллизации) на воздухе при различных температурах $\left(T_{a n n}\right)$ от 580 до $650^{\circ} \mathrm{C}$. В стехиометрический состав мишени добавлялось $10 \mathrm{~mol} \% \mathrm{PbO}$ для восполнения потерь свинца в процессе кристаллизации фазы перовскита. Толщина осажденных пленок PZT составляла 0.5-1 $\mu \mathrm{m}$. В качестве верхних электродов также использовалась платина.

Процессы релаксации исследовались как при квазистатическом изменении внешнего электрического по- ля, так и в полях, соответствующих максимумам диэлектрической проницаемости на реверсивных зависимостях $(\varepsilon-V)$. Релаксационные зависимости емкости и диэлектрической проницаемости изучались с помощью измерителя иммитанса E7-20 на частоте $10 \mathrm{kHz}$ при измерительном напряжении $100 \mathrm{mV}$. Временные зависимости локального пьезоэлектрического отклика пленок PZT исследовались с использованием силовой микроскопии пьезоэлектрического отклика в контактной моде на сканирующей нанолаборатории Ntegra Prima (NT-MDT). Измерения проводились на частоте $150 \mathrm{kHz}$ при приложении переменного напряжения с амплитудой $5 \mathrm{~V}$ и приложении на кантилевер NSG30/Pt постоянного смещающего напряжения $\left(U_{=}\right)$в диапазоне от 10 до $30 \mathrm{~V}$. При этом фиксировались механические деформации, возникающие в результате данного воздействия, и их изменения во времени. Релаксационные зависимости пироэлектрического тока при переключении пленок PZT в постоянных электрических полях изучались динамическим методом исследования пироэффекта с использованием модулированного импульсами прямоугольной формы лазерного ИК-излучения $(\lambda=980 \mathrm{~nm})$.

На рис. 1, $a$ представлены реверсивные зависимости диэлектрической проницаемости $\varepsilon(V)$ для пленок PZT, отожженных при различных температурах: кривая 1 соответствует температуре $T_{a n n}=580^{\circ} \mathrm{C}, 2-600^{\circ} \mathrm{C}$, $3-650^{\circ} \mathrm{C}$. Асимметрия максимумов $\varepsilon(V)$ характеристик по оси абсцисс свидетельствует о наличии в пленках внутреннего поля, определяющего направле- 

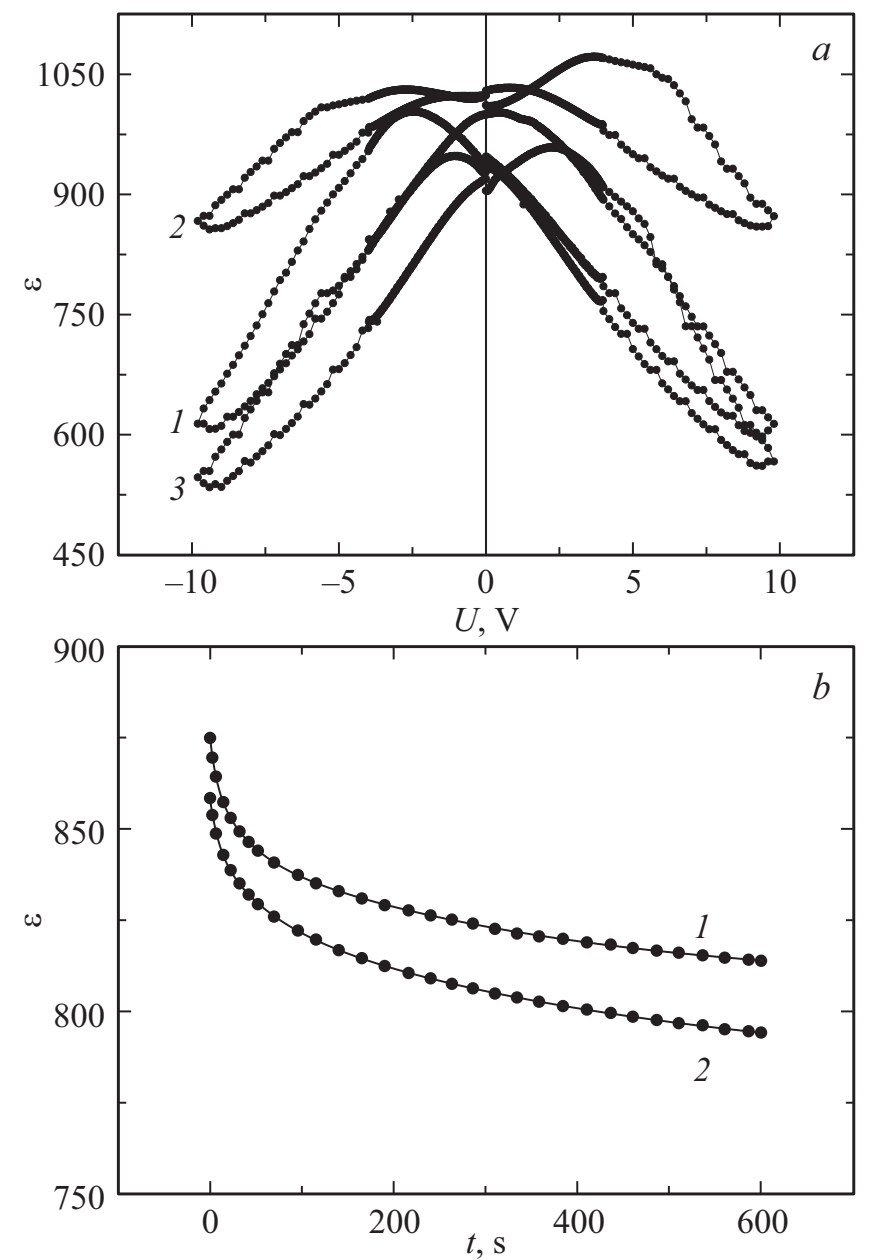

Рис. 1. (a) Реверсивные зависимости диэлектрической проницаемости для пленок РZT, отожженных при $T_{a n n}=580^{\circ} \mathrm{C}$ (кривая 1), $600^{\circ} \mathrm{C}(2), 650^{\circ} \mathrm{C} \quad(3)$; $(b)$ релаксационные зависимости диэлектрической проницаемости в пленках PZT $\left(T_{a n n}=580^{\circ} \mathrm{C}\right)$. Кривая 1 соответствует случаю: $E_{\text {pol }} \uparrow \uparrow P_{\text {self }}$, кривая $2-E_{\text {pol }} \downarrow \uparrow P_{\text {self }}$. Точки - экспериментальные результаты, сплошные линии - их аппроксимация.

ние вектора самополяризации $\left(P_{\text {self }}\right)$. Увеличение $T_{a n n}$ приводит к изменению направления внутреннего поля и ориентации $P_{\text {self }}$ на противоположное: если при $T_{a n n}=580^{\circ} \mathrm{C}$ вектор $P_{\text {self }}$ в пленке направлен в сторону подложки (рис. $1, a$, кривая 1 ), то при $T_{a n n}=650^{\circ} \mathrm{C}-$ к ее свободной поверхности (рис. 1, а, кривая 3) [8]. При $T_{a n n}=600-620^{\circ} \mathrm{C}$ на реверсивной зависимости наблюдается нескольких максимумов диэлектрической проницаемости (рис. 1, $a$, кривая 2), наличие которых может свидетельствовать о существовании в этих пленках некоторого промежуточного состояния, определяющего переход от одного направления вектора $P_{\text {self }}$ к другому [8].

В полях, соответствующих максимумам $\varepsilon(V)$ характеристик и различным направлениям поляризующего поля $\left(E_{p o l}\right)$ относительно направления вектора самополяризации, исследовались временные зависимости ди- электрической проницаемости, типичные кривые которых представлены на рис. $1, b$. Кривая 1 соответствует случаю, когда вектор поля $E_{p o l}$ коллинеарен и сонаправлен вектору $P_{\text {self }}\left(E_{\text {pol }} \uparrow \uparrow P_{\text {self }}\right)$, а кривая $2-$ когда их направления антиколлинеарны $\left(E_{p o l} \downarrow \uparrow P_{\text {self }}\right)$. Обе экспериментальные зависимости (точки) хорошо аппроксимируются суммой 3-х экспонент (сплошные кривые) и характеризуются тремя временами релаксации $\tau_{1}, \tau_{2}$ и $\tau_{3}$, которые, в свою очередь, определяют разные механизмы диэлектрической релаксации. Расчет времен релаксаций показал, что в полях, совпадающих с направлением самополяризации, релаксационный процесс осуществляется быстрее. Средние значения времен релаксации диэлектрической проницаемости при различных температурах отжига поликристаллических пленок PZT для случаев, когда поляризующее поле сонаправлено $\left(E_{p o l} \uparrow \uparrow P_{\text {self }}\right)$ и противоположно направлено вектору самополяризации $\left(E_{n} \downarrow \uparrow P_{\text {self }}\right)$, представлены в таблице.

Время $\tau_{2}$, значение которого составляет $\sim 30-90 \mathrm{~s}$, в соответствии с работами [3-5], характеризует инжекционный механизм заполнения ловушечных уровней в запрещенной зоне сегнетоэлектрика.

Более длинную релаксацию, определяемую сотнями секунд $\left(\tau_{3}\right)$, авторы работ связывают с миграционной максвелл-вагнеровской релаксацией $[3,4,6]$, вызванной транспортом кислородных вакансий, наличие которых обусловлено присутствием включений свинца в виде его оксидов - $\mathrm{PbO}$ и доокислением до $\mathrm{Pb}_{3} \mathrm{O}_{4}$ и $\mathrm{PbO}_{2}$ [7-10].

Однако в настоящее время нет единого мнения о природе процесса релаксации со временем $\tau_{1}$, величина которого определяется единицами секунд. Авторы работ $[5,6]$, исследуя ток при локальном переключении поликристаллических пленок РZT, связывают времена релаксации величиной от $0.1 \mathrm{~s}$ и более с переходными токами, обусловленными перезарядкой ловушек, локализованных на боковых границах зерен. Временная релаксация интегрального диэлектрического отклика конденсаторных структур на основе пленок PZT со временем $\tau_{1}$, на наш взгляд, является проявлением доменного вклада в процесс переполяризации. В ряде работ, например в [11,12], показано, что процесс переключения сегнетокерамики на основе PZT обусловлен наличием доменов в зернах. При этом процесс переключения, в основном,

Влияние температуры отжига образцов и ориентации внешнего электрического поля на времена релаксации, полученные из анализа временных зависимостей диэлектрической поляризации

\begin{tabular}{c|r|r|r|r|r|r|r|r}
\hline \multirow{2}{*}{$\begin{array}{c}\text { Времена релаксации } \\
\text { диэлектрической } \\
\text { проницаемости }\end{array}$} & \multicolumn{6}{|c}{ Температура отжига, $T_{\text {ann }},{ }^{\circ} \mathrm{C}$} \\
\cline { 2 - 8 } & 580 & 600 & 620 & 650 & 580 & 600 & 620 & 650 \\
\cline { 2 - 9 }$E_{\text {pol } \uparrow \uparrow} P_{\text {self }}$ & \multicolumn{5}{|c|}{$E_{\text {pol }} \downarrow \uparrow$} & $P_{\text {self }}$ \\
\hline$\tau_{1}, \mathrm{~s}$ & 7 & 3 & 10 & 11 & 10 & 5 & 12 & 13 \\
$\tau_{2}, \mathrm{~s}$ & 35 & 70 & 70 & 60 & 55 & 90 & 75 & 85 \\
$\tau_{3}, \mathrm{~s}$ & 350 & 280 & 480 & 450 & 460 & 450 & 640 & 600
\end{tabular}



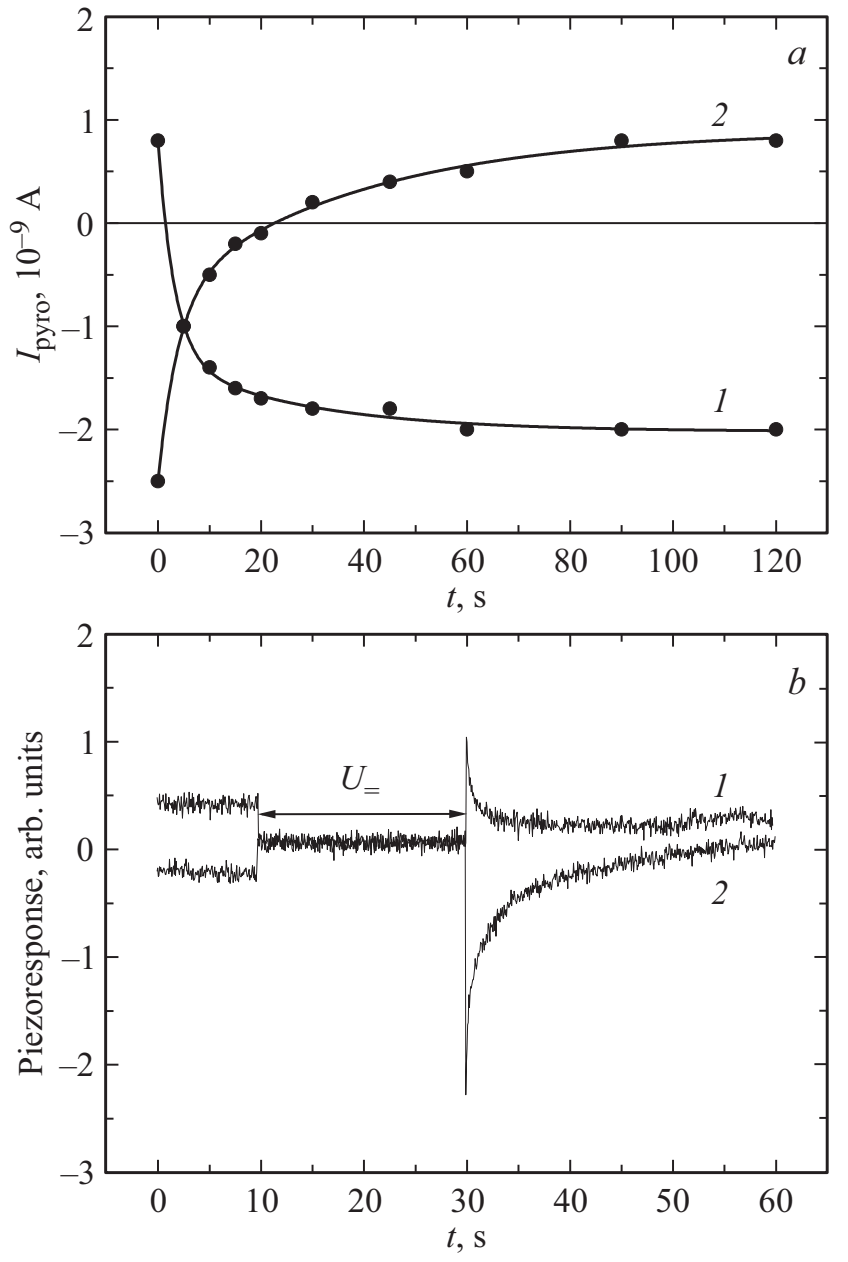

Рис. 2. (а) Релаксационные зависимости пиротока при переполяризации пленки PZT в поле $E_{p o l}=50 \mathrm{kV} \cdot \mathrm{cm}^{-1}$ и (b) релаксационные зависимости пьезоотклика при $U=30 \mathrm{~V}$. Кривые 1 соответствуют случаю сонаправленности коллинеарных векторов $\left(E_{p o l} \uparrow \uparrow P_{\text {self }}\right)$, кривые 2 - их антиколлинеарности $\left(E_{\text {pol }} \downarrow \uparrow P_{\text {self }}\right)$.

определяется зарождением и ростом антипараллельных доменов в кристаллитах [11]. В работе [13] обнаружено, что в тетрагональных пленках PZT приложение внешнего смещающего напряжения разных знаков приводит к движению доменных стенок. Несмотря на то, что исследуемые поликристаллические пленки PZT имеют мелкозернистую структуру, именно время релаксации $\tau_{1}$ является интегральной характеристикой процесса зарождения доменов новой (объемной) реориентации вектора сегнетоэлектрической поляризации в различных кристаллитах, находящихся под электродом при подаче на него постоянного электрического поля. Однако в объеме пленки за счет наличия несегнетоэлектрических фаз и нарушения кристаллической структуры (прослойки оксида свинца, границы зерен, ростовые дефекты и т.д.) процесс переключения поляризации может затягиваться на секунды.
О вкладе доменного механизма в процесс переполяризации свидетельствуют и результаты дополнительных исследований релаксационных процессов пиро- и пьезоэлектрических откликов (рис. 2, $a$ и, $b$ соответственно). При переключении пленки в постоянном поле $\sim 50 \mathrm{kV} / \mathrm{cm}$, приложенном в направлении $E_{\text {pol }} \uparrow \uparrow P_{\text {self }}$, релаксация пироотклика во времени аппроксимируется суммой двух экспонент и происходит со временами релаксации $\tau_{1}$ и $\tau_{2}$, величины которых соответственно равны 3 и $28 \mathrm{~s}$. Меньшее из этих значений определяет процесс реориентации сегнетоэлектрической поляризации (рис. 2, a, кривая 1), который проявляется сначала в уменьшении величины пиротока с последующим его ростом в противоположном направлении. В направлении $E_{\text {pol }} \downarrow \uparrow P_{\text {self }}$ процесс переключения затягивается, и времена релаксации увеличиваются до 4 и $40 \mathrm{~s}$, соответственно для $\tau_{1}$ и $\tau_{2}$, рис. $2, a$, кривая 2. Аналогичные результаты получены и для релаксационных зависимостей пьезоотклика, рис. $2, b$. Времена релаксации, определенные из аппроксимируемых экспоненци-
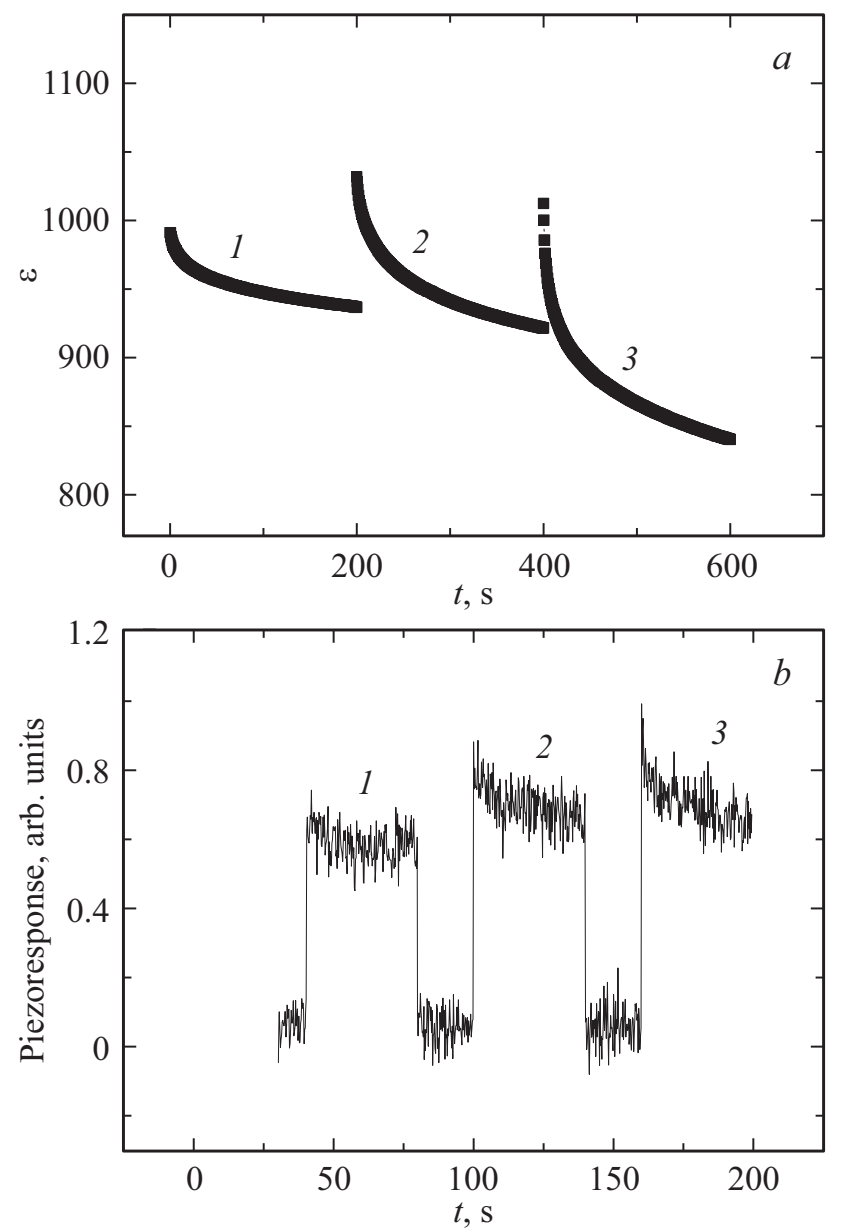

Рис. 3. (а) Релаксационные зависимости диэлектрической проницаемости для разных поляризующих полей $-E_{p o l}$ : $20(1), 60(2), 100 \mathrm{kV} \cdot \mathrm{cm}^{-1}(3)$ и (b) релаксационные зависимости пьезоотклика при различных приложенных напряжениях $U=10(1), 20(2), 30 \mathrm{~V}(3)$. 


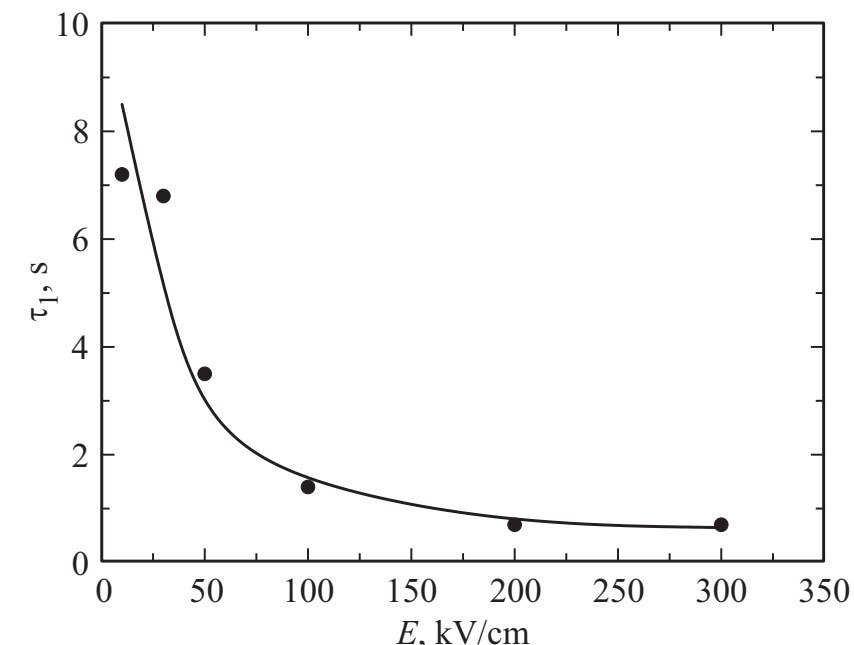

Рис. 4. Зависимость средних значений времен релаксации $\tau_{1}$ от величины постоянного поляризующего поля.

альными функциями зависимостей, близки по значениям временам $\tau_{1}$, определенным из релаксационных зависимостей диэлектрической проницаемости, и равны $\sim 3 \mathrm{~s}$ при сонаправленности коллинеарных векторов поля и самополяризации, и $\sim 4 \mathrm{~s}$ - при их антипараллельности. Видно, что и в этом случае релаксационные процессы, сопровождающие переключение, в направлении самополяризации проходят быстрее.

При квазистатическом изменении величины постоянного поля аппроксимация релаксационных зависимостей диэлектрической проницаемости (рис. 3,a) и пьезоотклика (рис. 3,b) показала, что в случае подачи на пленку поляризующего поля, превышающего коэрцитивное, релаксационные процессы характеризуются только одним временем релаксации $\tau_{1}$, а переключение пленки происходит быстрее, рис. 4. Как видно из рис. 4, время релаксации $\tau_{1}$ экспоненциально зависит от величины поляризующего поля, что, по всей видимости, свидетельствует о его непосредственной связи с доменным вкладом в процесс переполяризации. Таким образом, результаты релаксационных процессов в квазипостоянных электрических полях, представленные в настоящей работе и полученные из данных временных зависимостей диэлектрической проницаемости, пиро- и пьезоэлектрического откликов, свидетельствуют в пользу доменного механизма релаксации сегнетоэлектрической поляризации в поликристаллических тонких пленках PZT со временем релаксации $\tau_{1}$.

Анализ величин времен релаксации при переключении пленок PZT показал, что они оказались зависимыми не только от направлений векторов поляризующего поля и самополяризации, от величины самого поля, но и от температуры отжига (рис. 5). По мнению [14], последнее может быть связано со сменой местоположения зарождения фазы перовскита.
Аномально низким значениям $\tau_{1}$ соответствует $T_{a n n}=600^{\circ} \mathrm{C}$, при которой зарождение и рост фазы перовскита вблизи свободной поверхности пленки сменяется аналогичным процессом вблизи ее нижнего интерфейса. Это приводит, по мнению $[8,13]$, к локали-
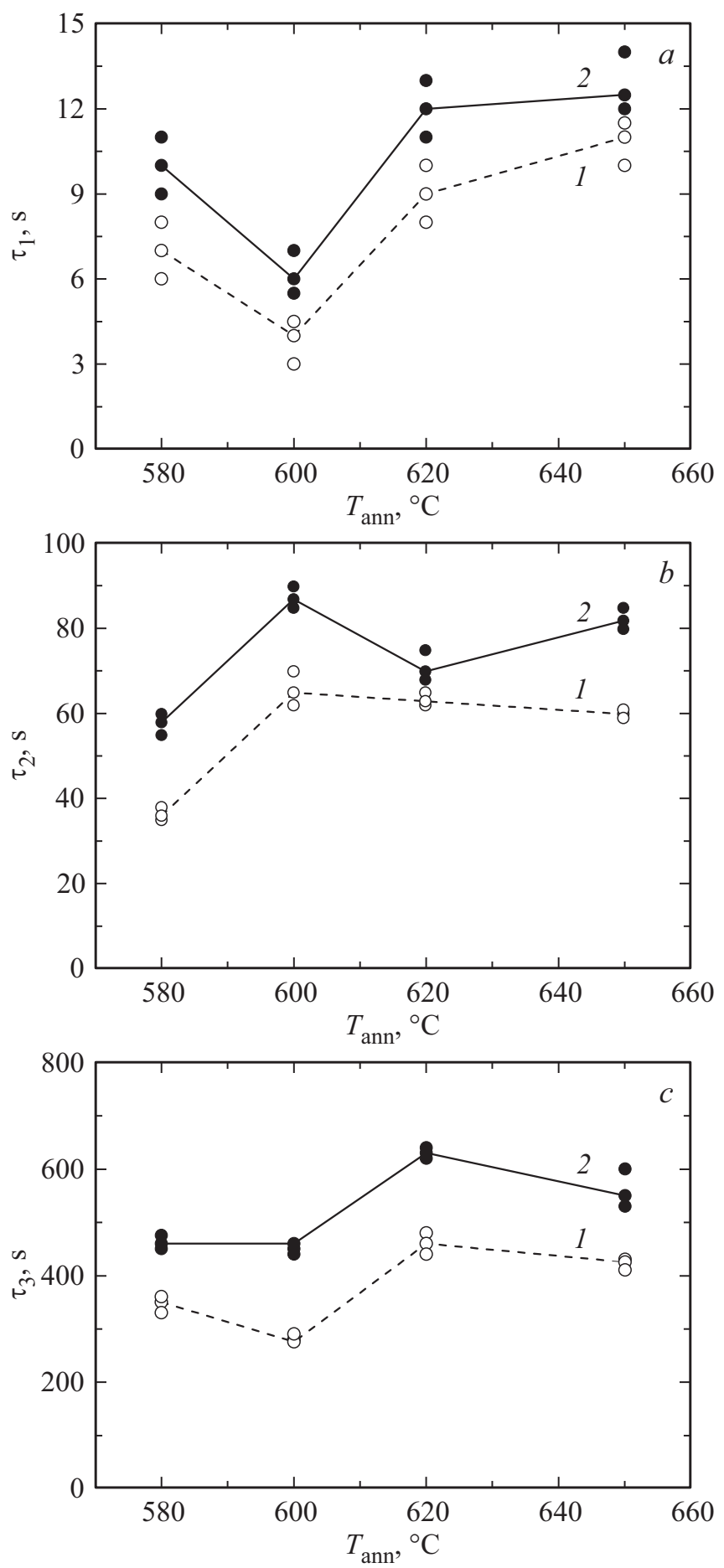

Рис. 5. Зависимости значений времен релаксации диэлектрической проницаемости от температуры отжига $T_{a n n}$ поликристаллических пленок РZT. Кривые 1 соответствуют случаю сонаправленности коллинеарных векторов $\left(E_{\text {pol }} \uparrow \uparrow P_{\text {self }}\right)$, кривые 2 - их антиколлинеарности $\left(E_{p o l} \downarrow \uparrow P_{\text {self }}\right)$. 
зации областей оксида свинца одновременно вблизи обоих (верхнего и нижнего) интерфейсов пленки и интегральному уменьшению избыточного оксида свинца, благодаря чему процесс релаксации доменной структуры происходит быстрее. Увеличение времени релаксации $\tau_{1}$ на рис. 5,a при меньших и больших значениях $T_{a n n}$ можно связать с пиннингом доменных границ, поскольку оно сопровождается ростом величины внутреннего поля, вызванного сформированным объемным зарядом вблизи нижнего или верхнего интерфейсов. Предполагается, что при дальнейшем увеличении $T_{a n n}$ содержание избыточного свинца и величина объемного заряда должны снижаться $[14,15]$.

Зависимость времен релаксации $\tau_{2}$ и $\tau_{3}$ от температуры отжига также во многом определяется наличием и перераспределением избыточного свинца в пленке. C ростом температуры отжига область существования пространственного заряда уменьшается. Тем самым создаются условия для дополнительной инжекции зарядов в пленку, что приводит к увеличению времени релаксации $\tau_{2}$. В присутствии меньшей области объемного заряда и уменьшения избыточного оксида свинца, что соответствует $T_{a n n}=600^{\circ} \mathrm{C}$, уменьшается концентрация зарядовых вакансий, что может вести к меньшим временам релаксации $\tau_{3}[14,15]$.

Таким образом, изучение процессов релаксации в пленках PZT, связанных с переполяризацией в квазистационарных электрических полях, показало, что времена релаксации, характеризующие разные релаксационные механизмы, определяются направлением самополяризации, зависят от величины поля переполяризации и температуры отжига.

\section{Конфликт интересов}

Авторы заявляют, что у них нет конфликта интересов.

\section{Список литературы}

[1] К.А. Воротилов, В.М. Мухортов, А.С. Сигов. Интегрированные сегнетоэлектрические устройства. Энергоатомиздат, М. (2011). 175 c.

[2] J.F. Scott. Science 315, 954 (2007).

[3] Y. Podgorny, K. Vorotilov, AIP Advances 6, 095025 (2016).

[4] Ю.В. Подгорный, К.А. Воротилов, А.С. Сигов, А.Н. Ланцев. Фундаментальные проблемы радиоэлектронного приборостроения 16 (2), 237 (2016).

[5] Л.А. Делимова, Е.В. Гущина, В.С. Юферев, И.В. Грехов. ФTT 56, 2366 (2014).

[6] L.A. Delimova, E.V. Guschina, D.S. Seregin K.A. Vorotilov, A.S. Sigov. J. Appl. Phys. 121, 224104 (2017).

[7] L. Delimova, E. Guschina, N. Zaitseva, S. Pavlov, D. Seregin, K. Vorotilov, A. Sigov. J. Mater. Res. 32, 1618 (2017).

[8] А.Г. Канарейкин, Е.Ю. Каптелов, С.В. Сенкевич, И.П. Пронин, А.Ю. Сергеенко, О.Н. Сергеева. ФТТ 58, 2242 (2016).

[9] И.П. Пронин, Е.А. Тараканов, Е.Ю. Каптелов Т.А. Шаплыгина, В.П. Афанасьев, А.В. Панкрашкин. ФТТ 44, 739 (2002).
[10] И.Ю. Тентилова, С.А. Кукушкин, Е.Ю. Каптелов, И.П. Пронин, В.Л. Уголков. Письма в ЖТФ 37, 37 (2011).

[11] Г.М. Акбаева, В.З. Бородин. ФТТ 57, 501 (2015).

[12] O.V. Malyshkina, E.V. Barabanova, A.I. Ivanova, A.V. Daineko \& V.A. Golovnin. Ferroelectrics 475, 82 (2015).

[13] M.-G. Han, M.S.J. Marshall, L. Wu, M.A. Schofield, T. Aoki, R. Twesten, J. Hoffman, F.J. Walker, C.H. Ahn, Y.-N. Zhuet. Nature Commun. 5, 4693 (2014).

[14] В.П. Пронин, С.В. Сенкевич, Е.Ю. Каптелов, И.П. Пронин. ФТT 55, 92 (2013).

[15] В.В. Иванов, Е.Н. Голубева, О.Н. Сергеева, Г.М. Некрасова, И.П. Пронин, Д.А. Киселев. Физико-химические аспекты изучения кластеров, наноструктур инаноматериалов. Межвуз. сб. науч. тр. Тверской гос. ун-т, Тверь 10, 270 (2018).

Редактор К.В. Емщев 